Le tuyau va en s'élargissanl progressivement, depuis une section circulaire de 18 pieds de diamètre au sortir de la turbine, jusqu'à une section oblongue composéc de deux demi-cercles de 22 pieds 8 pouces de diamètre, réunis par un rectangle de 17 pieds 6 pouces de large. Sa longucur est de 60 pieds. La vitesse de l'eau va en diminuant, depuis x3 pieds à la sortie de la turbine jusqu'à 4 pieds à la sortie du tuyau d'aspiration.

Le distributeur de la turbine est constitué par 20 vanneltes mobiles (guide vanes) en acier fondu, longues de 6 pieds 8 pouces $3 / 4$ et larges de 3 pieds 4 pouces. Ces vanettes pivottent chacune autour d'un axe vertical, entraînées qu'elles sont par une couronne horizontale (rocker ring) qui tourne d'un certain angle concentriquement à l'arbre de la turbine, et dont le mouvement est provoqué par deux servomoleurs à huile sous pression, dont l'un (governor cylinder) est visible sur la coupe verticale ci-jointe.

Le régulateur à boules et la pompe de circulation d'huile de ces scrvo-moteurs sont commandés par un axe horizontal (shaft to governor) qui reçoit son mouvement de l'arbre de la turbine par l'intermdéiaire d'un engrenage d'angle. La pompe, du type triplex, peut donner une pression de 200 livres par pouce carré (I/4 $\mathrm{kgs}$ par $\mathrm{cm}^{2}$ ). En outre de cette alimentation directe en huile des régulateurs, il existe une source auxiliaire spéciale qui est capable d'alimenter à la fois trois groupes électrogènes.

Le poids de la partic tournante, induit de l'alternateur et roue de la lurbine, est de 2.75 tonnes. Il est équilibré en partie par la poussée de l'eau sortant de la roue mobile, et en partie par un palier de butée à huile et à roulcaux. En marche normale, les rouleaux ne supportent aucune charge, mais si, pour une cause quelconque, la pression d'huile vient a baisser, ils sont prês à entrer en fonction, immergés dans leur bain d'huile.

En outre des 15 grosses turbines précitées, on installe actuellement deux turbinés de $2200 \mathrm{HP}$, destinées à actionner deux dynamos à courant continu pour l'excitation des alternateurs ct la commande de divers appareils nécessitant ct: genre de courant.

Le dernier tronçon du barrage a été complété le $x 6$ octobre dernier, et l'ensemble des travaux sera bientôt terminé. On compte mettre l'usine en marche dans le courant de l'année I 9 I 3 .

H. B.

\section{LA TERRE : MESURE DE LA SURFAGE ET DE SON RELIEF}

Discoụrs d'inauguration du $4 \mathrm{I}^{\mathrm{e}}$ Congrès de l'Association Française pour l'Avancement des Sciences, prononcé à Nìmes le I er août Ig I 2 , par M. Lallemand, inspecteur général des Mines, directeur du service du Nivellement général de la France, me mbre de l'Institut président de l'Association Française.

Pour me conformer à la tradition, je vais, dans un tableau rapide, retracer l'évolution, depuis son origine, de la branche de recherches à laquelle j'ai voué lc meilleur de mon activité : je veux dire la plus ancienne et la mère des Sciences, la Géométrie, prise dans sa plus large acception, qui est la " mesure de la Terre ".

Tout en m'excusant par avance de l'aridité du sujet, je vais brièvement passer en revue, dans son histoire, dans son état présent et ses applications, la détermination de la forme et des dimensions du globe, la figuration des accidents naturels et artificiels de sa surface, enfin la mesure de son relief.

\section{I. - La Fonde et les Dinensions de Globe}

A. - La Terre sphérique. - Pour les Grecs, au lemps d'llomère, soil dix siècles avant notre ère, la Terre étail un disque plat, sur les bords duquel reposail une immense voûte, le firmament, supportant les ćtoiles.

Ia disparition successive de la coque, d'abord, puis des mâls d'un navire qui s'éloigne, l'élargissement progressif et la forme toujours circulaire de l'horizon quand on s'élève au-dessus d'une plaine ou de la mer, donnèrent ensuile à quelques philosophes l'intuition que, dans son ensemble, la Terre avait la forme d'une calotle bombée.

Mais pour arriver à la notion d'un globe isolé dans l'espace, il fallut l'observation du cercle d'ombre projelé par la Terre sur le disque de notre salcllite, dans les éclipses de Lune; il fallut surtout le fail, qualifié d'invraisemblable par Ilérodote, que, contrairement à la règle invariable de nos régions, où le Solcil, à midi, se montre toujours à gauche quand on regarde l'ouest, des Phéniciens, dans un voyage de circumnavigation autour de l'Afrique, effeclué six siècles avant Jésus-Christ, avaicnl, au contraire, en doublant, vers l'oucst, l'acluel Cap de Bonne-Espérance, obscrvé, à midi, le soleil à leur droile.

La Terre étant reconnue ronde, pour en savoir le lour if suffisait de mesurer une fraction connue de la circonférence.

Deux sièces avant notre ère, un astronome grec, Eralosthène, avait obscrvé qu'à Syène, dans la IIaule-Egyple, lors du Solstice d'été, le Soleil éclaire les puits jusqu'au fond, tandis qu'à Alexandric, ville situéc au Nord de la première, sa direction, à la même époque, forme, à midi, avec le fil à plomb, un angle égal à la cinquantième parlic du ccrcle. Multipliant dès lors par cinquante la distance des deux villes. connue d'après le cadastre, Eráosthène en déduisil une valleur remarquablement approchéc de la circonférence terrestre.

Entre celte mélhode fort simple el celles employées depuis, pour celte même mesure, il n'y a d'autres différences que la perfection des instruments et la rigucur des observations.

Après la ruine de la civilisalion gréco-romaine el duranl tout le moyen-âge, la doctrine de la sphéricilé de la Terre re-. tombe dans l'oubli. Aux yeux de Saint-Auguslin, l'existence des antipodes n'est qu'une fable ridicule. Pour faire accepter à nouveau l'idée d'une Terre ronde, il ne faut rien de moins que la découverte de l'Amérique par Christophe Colomb.

En ${ }_{1528}$ seulement, soil 17 siècles après Eratosthène, au moyen d'un compleur de tours, précurseur du laximètre, adapté à la rouc de sa carriole, le médecin Fernel mesure la route, à peu près droilc el dirigée Nord-Sud, qui relic Paris à Amiens. De la différence connue des latitudes de ces deux villes, il conclut une nouvelle valeur de la circonférence terrestre, dont, par un heurcux hasard, l'erreur relative n'est que d'un millième.

Mais les mesures directes étant le plus souvent impossibles à cause des accidents du terrain, le hollandais Snellius, un siècle plus tard, imagine de jeter, entre les deux points extrêmes de l'arc à mesurer, une sorte de poutre géométrique, analogue aux poutres en treillis de nos modernes ponts métalliques, formée de triangles juxtaposés, dont on observe les trois angles et dont, par un autre enchaînement de triangles, on raltache l'un des côtés à une base rectiligne facilè à mesurer.

De la sorte, on a tous les éléments nécessaires pour calculer la longueur de la poutre entière. 
Sous le nom de "triangulation ", ce procédé, connu très probablement déjà des anciens Egyptiens, est universellement appliqué depuis Snellius.

B. - L'ellipsoïde terrestre. - Dès lors, les progrès se multiplient.

Vers la fin du XVII ${ }^{\circ}$ siècle, ayant mesuré la France dans toute sa hauteur, de Perpignan à Dunkerque, Cassini trouve, pour le degré de latitude, une longueur décroissant vers le Nord ; par suite, au lieu d'être parfaitement ronde, la Térre aurait la forme d'un œuf allongé vers les pôles.

Avec Newton et Clairaut, la théorie, pourtant, exigeait un globe aplati aux pôles et renflé à l'équateur. Pour vider la querelle, l'Académie des Sciences fait mesurer deux nouveaux arcs, l'un en Laponie, l'autre au Pérou. Cette fois, la théorie triomphe.

Et depuis lor's, les géodésiens couvrent de triangles le globe et mesurent partout des arcs de méridiens et de parallèles, à l'effet de délerminer, avec une précision croissante, l'aplatissement et le rayon équatorial de l'ellipsoüde terrestre, dont, en particulier dérive la valeur du mètre.

Parmi les plus importants de ces arcs figurent le grand arc méridien du Cap au Caire, dont les géodésiens anglais et allemands viennent d'entreprendre la mesure, et dans l'Amérique du Sud, amorce d'une grande chaîne qui rejoindra plus tard cclle du Nord, le petit arc de l'Equateur, récemment mesuré par une mission d'officiers français du service géographique de l'armée, sous le contrôle de l'Académie des Sciences.

C. - Le géoïde. - Mais bientôt, et ceci ne date pas d'un demi siècle, on conslale avec surprise qu'en maints endroits la verticale n'est pas perpendiculaire à la surface de l'ellipsoïde théorique. Cette surface, désormais appelée de géoïde, présenterait donc des saillies et des dépressions

El aussitôt les géodésiens de se mettre à l'œuvre pour mesurer ces bosses, dont plusieurs atteignent jusqu'à roo $\mathrm{m}$. d'élévation.

D'autre part, il y a une douzaine d'années, on s'apercevait que les pôles terrestres eux-mêmes, jusque-là regardés comme fixes, se déplacent à la surface du sol et subissent un mouvement oscillatoire de quelques mètres d'amplitude et d'environ 430 jours de période, faisant varier d'autant les latitudes géographiques ${ }^{(1)}$.

D. - Les déformations lentes et les marées de l'écorce terrestre. - Et ce n'est pas toul.

Notre planète rayonnant dans l'espace, le noyau central igné se refroidit et se contracte, et, dès lors, le géoïde, lui aussi, se déforme lentement dans le cours des siècles.

Tout dernièrement même, j'ai pu montrer qu'à l'instar des Océans, et du fail de la rolation diurne combinée avec l'attraction du Soleil et de la Lune, l'écorce lerrestre subit un soulèvement et un affaissement journalicrs, analogues au flux et au reflux de la mer.

Mais ce mouvement périodique, dont l'amplitude, à l'équatcur, atteint un demi-mètre, laisse intacle, en moyenne, la forme générale de la surface.

\section{II. - Les Accidents de la Surface terrestre ET SES Divisions territoriales \\ Cadastre et Gartes topograpiugues}

A. - Cadastre. - La forme générale du globe étant connue avec une suffisante approximation, il a fallu, dans tous

(1) Depuis rgoo, l'Association géodésique internationale poursuit, avec succès, l'étude de ce passionnant problème. les pays et pour les besoins de la civilisation, se préoccuper d'y situer, à leur juste place, les accidents qui émaillent la surface terrestre : fleuves, montagnes et cilés, voies de communication, divisions administratives et limites de propriétés.

En vue, notamment, d'une plus équitable répartition de l'impôt foncicr, comme aussi pour mieux fixer les limites des héritages, tous les peuples cultivés, à commencer par les Egyptiens, se sont efforcés de créer, sous la forme de plans cadastraux, une sorte d'état civil de la propriété foncière.

Pour atteindre ce but, le procédé le plus sûr et le plus simple à la fois consiste à couvrir le territoire d'un réseau de triangles, aux sommets desquels on rattache ensuite tous points intéressants du sol et jusqu'aux bornes des parcelles de champs.

Pour que les plans parcellaires de communes limitrophes puissent se raccorder entre eux, sans lacunes ni duplicatures, il faut qu'ils aient été ainsi rattachés à une triangulation générale préalable du territoire. C'est là une nécessité primordiale universellement reconnue, une règle partout observée, sauf, hélas, en France, où, dès $18 \mathrm{r} 7$, une Commission officielle, comptant parmi scs membres les académiciens et les astronomes Laplace, Puissant, Delambre, Poisson et Mathieu, signalait, chez les agents du Cadastre chargés de la triangulation, une complète inaptitude à exécuter les plus simples des opérations à eux confiées... et l'ignorance absolue de toutes notions techniques ou mathématiques.

Malgré les objurgations réitérées de toutes les autorités en la matière et de tous les corps savants, notre ancien cadastre, commencé en r $79^{\circ}$ el non encore achevé, n'a jamais été rattaché à la grande triangulation de l'élat-major.

A raison de cette lacune initiale, jointe à l'absence de tenue à jour des plans, celte œuvre gigantesque, qui a coûté des centaines de millions, doit être aujourd'hui complètement refaite.

Telle est la fâcheuse conclusion d'une enquête à cet égard, faite, il y a vingt ans, par la Commission extraparlementaire du Cadastre, instituée à la suite de vœux pressants des communes et des Conseils généraux.

Après des études et des essais nombreux, un programme de réfection avait été dressé par cette Commission.

Outre l'achèvement de la triangulation générale par le service géographique de l'Armée et le rattachement des nouvelles opérations cadastrales à cette triangulation, l'on prévoyait, dans ce projet, avec un large emploi de machines pour les calculs, l'intensive application du principe industriel de la division du travail. Pour lo rapport des plans, on renonçait au papier, substance déformable rt fragile, se prêtant mal aux opérations permanentes de mise à jour et ne permettant les reproductions quc sous la forme infidèle et coûteuse de photographies ou de copies à la main. A ce système suranné, l'on substituait la gravue sur zinc, directement exécutée à l'envers, ainsi que les écritures, par des spécialistes ; au moyen d'un simple passage à la presse, les planches ainsi obtenues et d'ailleurs faciles à tenir constamment à jour devaient ensuite fournir, à peu de frais, pour le public et les Administrations intéressées, un nombre illimité d'exactes reproductions des plans minutes.

A raison de ro francs en moyenne par hectare, soit 7 pour I ooo de la valeur vénale moyenne du sol, I 500 francs l'hectare, l'exécution de ce programme pour la France entière, devait coûter un demi-milliard environ.

En 1898 , une loi provisoire avait été votée, permettant aux communes d'obtenir, avec le concours financier de l'Etat et des Départements, la réfection de leur cadastre d'après ces 
principes nouveaux ; plusieurs centaines d'entre elles avaient déjà réclamé lc bénéfice de cette loi ; sur le dixième du terriloire, la triangulation générale avait été revisée el compléléc par le Service Géographique de l'Armée; des méthodes et des instruments perfectionnés avaient été créés, el une centaine d'élèves formés à leux emploi ; de divers pays élranger's, des missions d'ingénieurs et d'officiers venaient successivement s'initier à la pratique de ces nouveaux procédés, partout cilés comme des modeles. Bref, l'œuvre entrait dans l'ère de plein fonctionnement, lorsque brusquement, en 1907, sous prétexte d'économics, sans avoir consulté aucun des hommes des Comilés, ou des Services compétents, on arrêlait la revision de la triangulation générale ; puis, devant la soi-disant indifférence du public à l'achat des plans ${ }^{1}$ ), on supprimail à son tour la gravure sur zinc el l'on décapitait le scrvice en nommant percepteurs les plus habiles de ses agents, géomèlres ou graveurs, recrutés avec tant de peine el formés à si grands frais ; enfin l'on mellait à l'encan les travaux, désormais dépouillés des élémenls qui en doublaient la valeur.

L'unc après l'autre, soucicuses du bon renom scicnlifique de nolre pays, comme aussi de la marche logique el de l'économic des opérations, la Commission Centrale des travaux géographiques, le Bureau des Longitudes, la Commission géodésique française et l'Académic des Scicnces clle-même, protestaient contre ces mutilations, qu'à la tribune du Sénat, M. Boudenoot, auteur de la loi de $\mathrm{r} \& 98$, dénonçait à son tour comme un vérilable "sabotage adminisralif ".

Mais ce fut en vain.

Malgré les prélendues "simplifications" apportées au programme, un premier essai d'adjudication, en juillet r $9 \mathbf{I}$, échouait lamentablement. Pour un tiers seulement des Communes en cause, les travaux offerts trouvaient prencurs.

Une amnée après, malgré une majoration de 40 pour soo des larifs $\left(^{2}\right)$, ce qui porterail à 700 millions le devis tolal, une scconde lentative n'avait guère plus de succès.

Si ces mesures néfastes ne sonl pas rapportées, peut-être, finalement, au licu de 500 millions qu'eussent coûlé des plans finement gravés, faciles à reproduire exactement el à conserver, nous faudra-t-il payer le double pour do simples minutes sur papier, praliquement impossibles à lenix à jour, et pour de mauvaises copies lithographiques non cotées, n'offrant aucune garanlie certaine de conformité avec l'original.

B. - Cartes topographiques. - En assemblant leurs plans cadastraux sur un canevas déduil d'une triangulation générale, et en prenant du tout une image réduite, la plupart des Etats civilisés ont dressé une carte générale de leur territoire.

\footnotetext{
(1) Pour montrer ce que peut valoir ce prétexte, il suffit de citer l'exemple de deux pays où, depuis l'origine, les plans cadastraux sont reproduits et mis en vente et dont les habitants ne passent certes pas pour être plus cultivés que nos compatriotes.

En Italie, au 30 juin Igo3, on avait publié les plans complets de $955 \mathrm{com}-$ munes, occupant 32 . 26 planches, dont il avait été tiré 356.473 copies. Ia vente de ces copies, en progression croissante d'ailleurs, avait, dans la dernière année, rapporté $53.67^{2}$ francs.

En particulier, dans la province de Milan, les demandes avaient été si nombreuses qu'il avait fallu procéder à un nouveau tirage de 87 r feuilles de l'édition primitive épuisée.

En Bavière, d'autre part, où, depuis le début du XIXe siècle, les plans cadastraux sont lithographiés, et oû l'on a dépensé à cet effet plus de 4 millions et demi de francs, il est vendu chaque année environ 85.00o feuilles. Dans un rapport officiel, la Commission du Cadastre déclare que la collection des planches matrices constitue, pour le pays, un "trésor inestimable" !
}

(2) En y comprenant les dépenses de reproduction photozincographique des plans.
C'est ainsi, par cxemple, qu'a été obtemue chez nous liı belle Carte d'Etal-major au $80000^{\circ}$, gravéc de 1818 à i $88 \%$ et dont dérivent toules nos aulres cartes nationales.

De même, l'élablissement d'une nouvelle carle plus délaillée, à l'échelle du $50000^{\circ}$, était prévuc comme corollaire de la réfection de notre Cadastre.

En assemblant de façon analogue el réduisant ì plus petite échelle les cartes nalionales on oblient des cartes inlernationales ou mondiales.

La plus récente est la carte du monde an millionièno, dont en rgog, les principaux Ftals civilisés onl, d'un commun accord, arrêlé les cadres el le lype. L'exécution en est déjà commencéc.

Une ouvre analogue est la carte internalionale aćronantique à l'échelle du $200000^{\circ}$, dont les principes directeurs, calqués sur ceux de la carte an millioniène, ont recucilli, cn novembre rgr a à Turin, l'approbalion unanime du cinquième Conglès international d'aviation el, an mois de juin dernier, à Vienne (Autriche), celle de la Fédéralion aćronaulique internationale.

Sur l'inilialive du gouvernement français, une conférenec officiclle de délégués compétents des divers pays se réunira prochainement, à Paris, pour sanclionner ce projet.

\section{III. - Le Recief de la Surface teminestre}

L'écorce terrestre se trouve en partic recouverte par l'eau des Océans.

Lorsque, jetant les yeux sur une mappemonde, on examine la distribution des teres, on est lout d'aboted frapmí de la réparlition de colles-ci en 3 grandes masses effilées en pointes vers le Sud, savoir, le groupe des deux Améri-

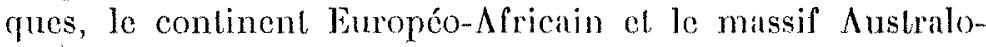
Asiatique, auxquels s'opposent respeclivement l'Océan lndien, le Pacifique el l'Allanlique. Ies récenles expédilions de Shackleton el d'Amundsen au pôle Sud, y ont en oulre démontré l'existence d'un vaste contincul, landis qu'au pôle Nord, Nansen a trouvé une mer libre el profonde.

Pour expliquer ces fails, W. Green, vers 188?, émil l'illóc que le noyau central fluide se refroidissanl, par l'effel du rayonnement dans l'espace, el se contractant plus que l'ćcorce, celle-ci, d'après la loi du moindre effort, devait tendre vers une forme dérivée du télraèdre, ou pyramide à quatre faces, qui est le solide régulier embrassant le plus petit volume sous une surface extéricure donnéc.

Dans ce solide, en effet, chayue pointe saillante a pour antipode une face plane.

Par la différence des vilesses de rolation du triple renllement boréal, d'une part, et de la pointe australe de la toupie terrestre d'autre part, Green a ćgalement expliqué la déviation sýstématique, vers l'Est, des trois arêtes dirigées vers celle pointe el la formation de celle suite de dépressions : fosse de la Méditerranćc, détroits de la Sonde ol mer des Antilles, qui entourent le globe el coupent en deux parties chacune de ces mêmes arêtes.

Mais ce n'est là qu'une très insuffisante définilion du relief terrestre.

Pour la plupari des travaux ayant le sol pour théatre, qu'il s'agisse d'établir une voie de communication ou une conduite d'eau, de créer un canal de drainage ou d'irrigation, d'apprécier le rendement possible d'une chule d'ean ou de construire un ouvrage de défense militaire, une connaissance plus précise el plus détaillée du relief est indispensable.

Suivant le cas, en effel, il faut reconnaître le parcours le 
plus avantageux au point de vue des dénivellations à franchir, calculer la profondeur des tranchées à ouvrir et la hauteur des remblais à élever, mesurer les pentes et les rampes de la nouvelle voie, etc.

Pour ces divers objets, on définit le relief, soit au moyen d'altitudes, qui sont respectivement les hauteurs des points du sol au-dessus de la surface de la mer, prise comme base de comparaison et prolongée par la pensée sous les continents, soit au moyen de courbes cotées, dites courbes de niveau, qui sont les lignes étagées qu'occuperait successivement le rivage des mers si, brusquement, leur niveau montait de ro, 20, rno ou 1 ooo mètres par exemple.

Reste à mesurer les altitudes et à fixer le tracé des courbes de niveau.

Pour cela, au moyen d'une suite de visées horizontales, faites sur des échelles ou mires disposées verticalement de distance en distance, on constitue, le long d'itinéraires convenables, l'équivalent d'une sorte de canal, dont on noterait successivement, pour les cumuler ensuite depuis l'origine, les hauteurs des écluses montantes et descendantes.

Vers r 855, un conducteur français des Ponts et Chaussées, Bourdaloue, a, le premier, pensé que si la planimétrie d'un vaste territoire doil avoir pour base un réseau de grands triangles, sur les sommets desquels s'appuie le relevé des points de détail, de même l'hypsométrie générale d'un grand pays doit reposer sur un réseau plus ou moins dense de lignes de nivellement, ou plutôt sur une série de réseaux, à mailles de plus en plus étroites, dont chacun s'appuie sur le précédent.

G'est ainsi qu'en France, de r855 à r863, a été constitué l€ premier réseau général de nivellement. Il avait 15000 kilomètres de développement.

Cet exemple a été suivi partout à l'étranger, si bien qu'à cette heure, si l'on mettait bout à bout les lignes de nivellement existant dans le monde, elles feraient plus de huit fois Je tour de la terre.

Devant l'ampleur et la précision croissantes des résultats obtenus autour de nous, l'Administration des Travaux Publics, dès $\mathrm{x} 884$, prenait le parti d'établir un nouveau réseau fondamental, sur lequel se grefferaient successivement plusieurs réseaux secondaires.

Le Réseau de base, à mailles de 400 à 600 kílomètres de tour, mesurant au total $\mathrm{r} 2$ ooo kilomètres de développement, était achevé en r 892 . Sa précision est telle qu'en revenant au point de départ, après avoir parcouru le tour de la France, soit 3908 kilomètres, on a pu retrouver, à 6 centimètres près, l'altitude initiale.

Le réseau de deuxième ordre, à peu près aussi tendu que le réseau fondamental, mais à mailles deux fois plus petites, est aussi terminé depuis 1898 .

Le réseau de troisiěme ordre dont les mailles n'ont plus que 60 à roo kilomètres de tour et dont la longueur atteindra $47000 \mathrm{kms}$, est lui-même aux neuf dixièmes achevé.

Enfin près du quart du réseau de $4^{\circ}$ ordre (mailles de ro à 20 kilomètres) est déjà nivelé.

La longueur totale des nivellements qui sillonnent, à cette heure, le territoire de la France, est d'environ roo ooo kilomètres.

En outre, depuis quelques années, dans les Alpes et les Pyrénées, ces travaux sont complétés par le relevé des profils en long de torrents, dont, par ailleurs, on a mesuré les débits, à l'effet de pouvoir ainsi dresser une statistique exacte des grandes forces hydrauliques disponibles dans cf; régions.

\section{$\star^{\star} \star$}

Cette rapide esquisse aura suffi, j'espère, à montrer quelle part prépondérante, notre pays, de tous temps, a pris dans les études concernant la forme de la Terre, les accidents de sa surface et la mesure de son relief.

En matière de géodésie, le cadastre ou le nivellement, comme en tant d'autres domaines, la France a été l'initiatrice et l'ouvrière du progrès.

Au début du siècle dernier, la Science lui était redevable de ces mesures d'arcs méridiens, d'où, à proprement parler, la Géodésie a pris naissance, et dont est sortie l'œuvre magistrale du Système métrique.

Tout récemment encore, à l'Equateur, ai-je dit, nos officiers répétaient, avec une précision jusque-là inconnue, la mesure d'un de ces arcs.

G'est en France qu'esi né l'art des nivellements généraux, et l'étranger, en adoptant nos méthodes et nos in truments, n'a cessé de rendre hommage à leur supériorité.

En matière de Cadastre aussi, nous avons autrefois occupé une place d'élite.

Derrière les aigles victorieuses des armées impériales, dans Je Palatinat, les Flandres, la Toscane, on voyait, il y a plus d'un siècle, les géomètres français dresser le cadastre des territoires conquis ; les plans levés par eux, longtemps regardés comme des modèles, sont encore, en maints endroils, les seuls employés.

Puisse, à cet égard, l'éclipse actuelle n'être que passagère, et la France reprendre bientôt le poste d'avant-garde qu'elle n'aurait jamais dû quitter.

\section{FIXATION DE L'AZOTE PAR L'ALUMINE ET LE GARBONE}

Note présentée par S.-A. Tucken, et Henry Reav, au deuxième Congrès général de la Société Electrochimique américaine, à New-York (Septembre 1912).

L'objet de cette étude était de rechercher si l'azote se fixe sur un mélange chaud d'alumine et de carbone, ou sur les produits dérivés du chauffage de ce mélange, et d'étudier les meilleures conditions de réalisation de cette fixation.

Les auteurs désiraient en outre rechercher les conditions dans lesquelles l'ammoniaque peut être obtenue à partir des produits azotés résultant de celte réaction.

L'aluminium et l'azole formenl, dans certaines conditions, une combinaison de formule $\mathrm{AlAz}$ ou $\mathrm{Al}^{2} \mathrm{Az}^{2}$ qui est connue depuis longtemps ${ }^{1}$ ). L'azoture d'aluminium est décrit comme une poudre grisc amorphe. Il est décomposé en partie par l'eau, en donnant naissance à de l'ammoniaque, et cette réaction se réalise par l'exposition prolongée à l'air. La décomposition complète est obtenue par le chauffage dans une solution alcaline.

Ottokar SERper est vraisemblablement le premier qui considéra la possibilité d'appliquer la réaction des composés alumineux sur l'azote à la fixation de l'azote atmosphérique. Dans de nombreux brevets $\left(^{2}\right)$ pris aux Ftats-Unis et ailJcurs, il a revendiqué la fabrication de l'azoture d'aluminium, mais sans donner grands détails sur la nature et les

(1) Zcitschr. Anorg. Chem. 54, 522-7 (Fichte).

(2) Brevets U.S.A. : 888.044;867.615; $987.408 ; 996.032$.

Brevets allemands: $181.991 ; 181.992$ : $183.702 ; 216.746$.

Brevets anglais : 13.579 (1906); 15.996 (rgog); 25.141 (rgrr), 